\title{
Cohort study of adherence to adjuvant endocrine therapy, breast cancer recurrence and mortality
}

\author{
B Makubate ${ }^{1}$, P T Donnan ${ }^{2}$, J A Dewar ${ }^{3}$, A M Thompson ${ }^{3}$ and C McCowan ${ }^{\star 4}$ \\ ${ }^{1}$ Division of Population Health Sciences, Medical Research Institute, University of Dundee, Mackenzie Building, Kirsty Semple Way, \\ Dundee DD2 4BF, UK; ${ }^{2}$ Dundee Epidemiology and Biostatistics Unit, Division of Population Health Sciences, Medical Research \\ Institute, University of Dundee, Mackenzie Building, Kirsty Semple Way, Dundee DD2 4BF, UK; ${ }^{3}$ Dundee Cancer Centre, Clinical \\ Research Centre, University of Dundee, James Arrott Drive, Dundee DD1 9SY, UK and ${ }^{4}$ Robertson Centre for Biostatistics, Institute \\ of Health and Wellbeing, College of Medical, Veterinary and Life Sciences, University of Glasgow, Boyd Orr Building, Level 11, \\ Glasgow G12 8QQ, UK
}

Background: Adjuvant endocrine therapy is recommended for women with oestrogen receptor-positive breast cancer, but many women do not take the medication as directed and they stop treatment before completing the standard 5-year duration.

Methods: This retrospective cohort study conducted between 1993 and 2008 of all women with incident breast cancer, who are residing in the Tayside region of Scotland, examined adherence to prescribed adjuvant tamoxifen or aromatase inhibitors (Als). Survival analysis examined the effect of adherence on all-cause mortality, breast cancer death and recurrence, using linked prescribing, cancer registry, clinical cancer audit, hospital discharge and death records.

Results: A total of 3361 women with breast cancer were followed for a median 4.47 years (interquartile range (IQR) $=2.04-8.55)$. The median overall adherence was $90 \%(I \mathrm{QR}=90-100 \%)$, but the annual adherence reduced after a longer period from diagnosis. Low adherence of $<80 \%$ was associated with poorer survival (hazard ratios $=1.20 ; 95 \%$ confidence interval $=1.03-1.40, P=0.019$ ). There was no significant difference for low adherence over the treatment period and recurrence, or breast cancer death, but patients with high annual adherence for 5 years had better outcomes than those with 3 or less.

Conclusion: Low adherence to all adjuvant endocrine therapy for women with breast cancer, whether tamoxifen or Al, increases the risk of death.

Adjuvant endocrine therapy is recommended for a 5-year period for women with early breast cancer with oestrogen receptor (ER)or progesterone receptor-positive cancers (Scottish Intercollegiate Guideline Network Guidelines, 2005). Aromatase inhibitors (AIs) and tamoxifen have been shown to reduce recurrence and mortality in post-menopausal women, and tamoxifen has shown similar benefits for pre-menopausal and peri-menopausal women (Cuzick et al, 2010; Early Breast Cancer Trialists' Collaborative Group, 2011; van de Velde et al, 2011).
Both classes of drug are taken orally on a daily basis, but many women do not take their medication as prescribed, with a proportion stopping before completing the full 5-year course or others not taking a daily tablet on a regular basis (Ruddy et al, 2009). Stopping medication early relates to duration and persistence, or continuation of therapy. The former normally relates to the overall time a patient takes the medication, ignoring any breaks, whereas the latter measures the time from commencement of medication before a break of a pre-specified length.

*Correspondence: Dr C McCowan; E-mail: Colin.McCowan@glasgow.ac.uk

Received 6 December 2012; revised 20 February 2013; accepted 22 February 2013; published online 21 March 2013

(c) 2013 Cancer Research UK. All rights reserved 0007-0920/13 
Adherence is a measure of how well the patient takes the medication compared with the instructions given by the clinician, which is daily for endocrine therapy.

Studies using patients' pharmacy records have reported poor persistence with tamoxifen, with between one-third to half of the women stopping their medication early and up to $22 \%$ having stopped by 1 year (Barron et al, 2007; Ma et al, 2008; McCowan et al, 2008; Owusu et al, 2008; Narod, 2010; van Herk-Sukel et al, 2010). Other studies that examined patients taking tamoxifen or AIs reported $32 \%$ and $50 \%$, respectively, of patients stopping medication before 5 years (Hershman et al, 2010; van Herk-Sukel et al, 2010). Several of these studies and other work have shown an increased risk of mortality for patients who stop endocrine therapy early (Ma et al, 2008; McCowan et al, 2008; Yood et al, 2008; Hershman et al, 2011).

For adherence, self-reported adherence was higher than that seen using prescription data (Ziller et al, 2009). Studies that have examined prescribing records have reported between 19 and $28 \%$ of women having adherence below $80 \%$, and that although mean adherence is high, it falls progressively with each year from the date of diagnosis (Patridge et al, 2003; McCowan et al, 2008; Partridge et al, 2008; Hershman et al, 2010).

Previous work by the authors was the first to show an association between patients with low adherence to tamoxifen of $80 \%$ (or less) and increased risk of all-cause mortality (McCowan et al, 2008). Subsequent studies by other groups have confirmed this association, with one large US study showing an association for tamoxifen and AIs (Dezentje et al, 2010; Hershman et al, 2011).

This study aimed to extend that work (McCowan et al, 2008) by investigating adherence to adjuvant tamoxifen and AIs with a longer follow-up period of women with breast cancer, residing in Tayside, Scotland. The main objectives were to describe the use of endocrine therapy, including adherence and persistence for tamoxifen and AIs, and to investigate whether adherence to endocrine therapy was associated with all-cause and breast cancerspecific mortality, and recurrence after adjusting for clinical and demographic factors.

\section{MATERIALS AND METHODS}

Identification of study cohort. Women who were residents of Tayside, with a hospital discharge or cancer registry record for breast cancer from January 1993 to December 2008, or until death, were identified using previously described methods (McCowan et al, 2008). Prescribing, hospital discharge, cancer registry, cancer audit and General Registrar's Office death certificates were extracted, record-linked and anonymised for all women with breast cancer, who were resident for the entire study period or until death.

Study covariates. Date of diagnosis was recorded from either the Cancer Registry or the clinical audit records and age at diagnosis derived from this and the recorded date of birth. The duration between date of diagnosis and the first time the patient received treatment was calculated, and subsequent follow-up calculated from this initial date of treatment to the date of death or until 31 December 2008, the study cut-off point. Similarly, time taken to recurrence from start of treatment was calculated.

Deprivation was collected for patients using the Scottish Index of Multiple Deprivation based on home postcode (Scottish Government, 2012). Charlson's comorbidity index was derived for each patient using standard procedures and the ICD codes version 9 and 10 in the hospital admission records, and was augmented as previously described with prescribing records for specific medications (McCowan et al, 2008). Charlson's index scores were categorised into three groups with low (0-2), medium (3-5) and high $(6+)$ comorbidity.

The clinical characteristics of the cancer at diagnosis were recorded using clinical and pathological TNM categories (tumour stage, nodal stage and metastases).

Treatment adherence. Prescribing records for endocrine therapy (tamoxifen or AIs) and the number of days covered by each prescription, recorded from the number of dispensed tablets and the daily dose, were examined. Records for each individual patient were then used to calculate an adherence index across the entire duration of therapy, up to 5 years, based on the total days covered by all prescriptions and their duration of use. Patients with an adherence index less than $80 \%$, the cut-off value widely used in previous studies, were deemed to have low adherence (McCowan et al, 2008).

Patient medication use was also split into annual periods from initiating treatment until end of follow-up and adherence over each year calculated. The cumulative number of years, when the patient was deemed to have low adherence, were recorded for each annual period for a maximum of 5 years.

Persistence was calculated as the length of time from the first prescription to a break of at least 180 days before the completion of 5 years of therapy (Demissie et al, 2001). Patients were flagged as starting an endocrine therapy, and their dispensed prescribing were analysed to see whether they completed a 5-year course. Patients were flagged as stopping medication if they had no prescriptions for any endocrine therapy for a 6-month period and were censored if they died or were at the end of follow-up.

Outcome variables. The three main outcome measures were allcause mortality, breast cancer mortality and recurrence of breast cancer. Recurrence information was recorded from a case note review carried out on all identified patients for the period for 5 years after diagnosis. Date of death was obtained from the General Register Office death certificates, with the underlying cause of death examined and subsequently classed as breast cancer where appropriate.

Statistical analysis. Data were described as number of subjects (percentages) for categorical variables and mean (s.d.) for continuous variables. Where continuous variables did not follow a normal distribution, and tested using the Kolmogorov-Smirnov test for normality, the median and interquartile range (IQR) were reported. $\chi^{2}$-Tests for trend $\left(\chi^{2}\right.$-statistic, degrees of freedom, probability) were reported for differences in distribution of the population with $n$ categories. Cox proportional hazards models were employed to estimate hazard ratios (HRs) and 95\% confidence intervals (CIs) for each covariate for all-cause mortality, breast cancer mortality and recurrence. Patients were followed from commencement of endocrine therapy until time of death or the end of the study.

Multivariate results are presented as HRs and 95\% CIs. Covariates were included in the multivariate model if they were deemed to be of clinical significance. The proportional hazards assumption was assessed using the $\log (-\log$ (survival) $)$ plot, and those that failed the assumption or that were deemed to be time dependent were entered as continuous time-dependent covariates (Bradburn et al, 2003).

The study was granted approval from the Tayside Caldicott Guardian. The study used anonymous data with management and analysis performed in line with the Health Informatics Centre standard operating procedures, which are NHS Research Ethics Committee (REC)-approved; hence, specific REC approval for the project was not required. All statistical analyses were performed with IBM SPSS Statistics Version 17 (SPSS Inc. (2008)). 
Table 1. Demographic characteristics and presentation of cancer by use of adjuvant therapy in Tayside, Scotland $(n=4619)$

Patients with treatment (\%)

\begin{tabular}{|l|c|c|c|c|c|}
\hline & No treatment (\%) & Tamoxifen & Als only & $\begin{array}{c}\text { Mixed } \\
\text { No treatment vs treatment } \\
\left(\chi^{2} ; \text { d.f.; } \boldsymbol{P} \text {-value) }\right.\end{array}$ \\
\hline Numbers & $1258(27)$ & $2230(48)$ & $412(9)$ & $719(16)$ & -
\end{tabular}

\section{Age group}

\begin{tabular}{|l|c|}
\hline$<40$ & $74(6)$ \\
$40-49$ & $180(14)$ \\
$50-59$ & $293(23)$ \\
$60-69$ & $330(26)$ \\
$70-79$ & $227(18)$ \\
$80+$ & $151(12)$ \\
Unknown & $3(1)$ \\
\hline
\end{tabular}

\section{SIMD quintile}

\begin{tabular}{l|c|c|c|c|c}
\hline 1 & $137(11)$ & $307(14)$ & $56(13)$ & $90(12)$ & $115(16)$ \\
2 & $130(10)$ & $299(14)$ & $61(15)$ & $120(17)$ \\
3 & $194(15)$ & $362(16)$ & $63(15)$ & $2.7 ; 0.314$ \\
4 & $347(28)$ & $722(32)$ & $153(37)$ & - \\
5 & $220(18)$ & $500(22)$ & $77(19)$ & $161(22)$ \\
Unknown & $230(18)$ & $40(2)$ & $2(1)$ & $12(2)$ \\
\hline
\end{tabular}

\section{Charlson's index}

\begin{tabular}{|l|c|c|c|c|c|}
\hline $0-2$ & $368(29)$ & $650(29)$ & $132(32)$ & $230(32)$ & $277(38)$ \\
$3-5$ & $486(39)$ & $888(40)$ & $160(39)$ & $1.1 ; 2 ; 0.564$ \\
$6+$ & $404(32)$ & $692(31)$ & $120(29)$ & $212(30)$ \\
\hline
\end{tabular}

\section{Menopausal status}

\begin{tabular}{|l|c|c|c|c|c}
\hline Pre & $189(15)$ & $268(12)$ & $5(1)$ & $106(15)$ & $23(3)$ \\
Peri & $28(2)$ & $57(3)$ & $4(1)$ & $369(51)$ \\
Post & $646(52)$ & $914(41)$ & $301(73)$ & $2 ; 0.073$ \\
Unknown & $395(31)$ & $991(44)$ & $102(25)$ & - \\
\hline
\end{tabular}

\section{Tumour stage}

\begin{tabular}{|l|c|c|c|c|c|}
\hline 1 & $235(19)$ & $600(27)$ & $45(11)$ & $147(20)$ & $6.2 ; 3 ; 0.101$ \\
2 & $215(17)$ & $445(21)$ & $69(17)$ & $138(19)$ & - \\
3 & $59(4)$ & $91(4)$ & $12(3)$ & $34(5)$ & - \\
4 & $94(8)$ & $119(5)$ & $38(9)$ & $92(13)$ & - \\
Unknown & $655(52)$ & $975(43)$ & $248(60)$ & $308(43)$ & - \\
\hline
\end{tabular}

\section{Clinical nodes}

\begin{tabular}{|l|c|c|c|c|c|}
\hline 0 & $653(52)$ & $1386(62)$ & $137(33)$ & $421(59)$ & $11.7 ; 2 ; 0.003$ \\
1 & $147(12)$ & $194(9)$ & $59(14)$ & $21(3)$ \\
2 & $41(3)$ & $29(1)$ & $14(4)$ & $159(22)$ \\
Unknown & $417(33)$ & $621(28)$ & $202(49)$ & - \\
\hline
\end{tabular}

\section{Clinical metastases}

\begin{tabular}{|c|c|c|c|c|c|}
\hline $\begin{array}{l}\text { No } \\
\text { Yes } \\
\text { Unknown }\end{array}$ & $\begin{array}{l}755(60) \\
57(5) \\
446(36)\end{array}$ & $\begin{array}{c}1540(69) \\
50(2) \\
640(29)\end{array}$ & $\begin{aligned} 162 & (39) \\
17 & (4) \\
233 & (57)\end{aligned}$ & $\begin{array}{c}504(70) \\
40(6) \\
175(24)\end{array}$ & $\begin{array}{c}6.9 ; 1 ; 0.008 \\
- \\
-\end{array}$ \\
\hline \multicolumn{6}{|c|}{ Tumour grade } \\
\hline $\begin{array}{l}1 \\
2 \\
3 \\
\text { Unknown }\end{array}$ & $\begin{array}{c}67(5) \\
259(21) \\
593(47) \\
339(27)\end{array}$ & $\begin{array}{l}317(14) \\
827(37) \\
502(23) \\
584(26)\end{array}$ & $\begin{array}{c}27(7) \\
217(53) \\
141(34) \\
27(6)\end{array}$ & $\begin{array}{c}51(7) \\
296(41) \\
238(33) \\
134(19)\end{array}$ & $\begin{array}{c}266.8 ; 2 ;<0.0001 \\
- \\
- \\
-\end{array}$ \\
\hline \multicolumn{6}{|c|}{ ER status } \\
\hline $\begin{array}{l}\text { Positive } \\
\text { Negative } \\
\text { Unknown }\end{array}$ & $\begin{array}{l}412(33) \\
564(45) \\
282(22)\end{array}$ & $\begin{array}{c}1399(63) \\
165(7) \\
666(30)\end{array}$ & $\begin{array}{c}382(93) \\
8(2) \\
22(5)\end{array}$ & $\begin{array}{c}539(75) \\
28(4) \\
152(21)\end{array}$ & $\begin{array}{c}1021.6 ; 1 ;<0.0001 \\
- \\
-\end{array}$ \\
\hline
\end{tabular}

Abbreviations: $\mathrm{Al}=$ aromatase inhibitor; $\mathrm{ER}=$ oestrogen receptor; $\mathrm{SIMD}=$ Scottish Index of Multiple Deprivation. 


\section{RESULTS}

Overall, 4815 patients were identified with prescribing, hospital admission records for breast cancer surgery, cancer audit and cancer registry records over the 15 years between 1 January 1993 and 31 December 2008. Among these women, we excluded 195 women (4\%), who were known to have cancer before 1993, who were diagnosed with ductal carcinoma in situ or who received endocrine therapy more than 6 months before their diagnosis. This left 4619 patients for whom the median age at diagnosis was 63 years $(\mathrm{IQR}=52-74)$, and patients were followed for a total of 19890 person-years with a median follow-up of 4.47 years $(\mathrm{IQR}=2.04-8.55$ years). Sixty-nine per cent of women were followed up for at least 5 years or until death. Over the 15 years of the study period, there were 1621 (35\%) deaths, 1073 (23\%) attributed to breast cancer as the underlying cause of death and 761 (17\%) breast cancer recurrences were observed.

Effects of demographic factors on the use of the endocrine therapy. There were 1258 (27\%) women who did not receive adjuvant endocrine therapy. Older patients were more likely to use endocrine therapy $(P<0.001)$ and those with more advanced disease were less likely to do so (see Table 1). There were no significant differences in deprivation $(P=0.314)$, comorbidity scores at diagnosis $(P=0.564)$ or menopausal status $(P=0.073)$ between those who did and those who did not receive treatment.

The remaining analyses relate to the 3361 patients who commenced adjuvant endocrine medication. Five hundred and twelve patients (15\%) started on an AI, while 2849 (85\%) commenced on tamoxifen. Of the 512 patients started on an AI, 100 (20\%) switched treatments, while 619 (22\%) switched treatments from tamoxifen to AI. There were 1194 (36\%) of the 3361 women with breast cancer who died during the study.

Patients were generally highly adherent to their medication during the course of treatment with a median adherence of $90 \%$ $(\mathrm{IQR}=90-100 \%)$. However, looking at annual adherence over time from starting medication, there was a decrease in adherence: the median adherence was $90 \%, 82 \%, 77 \%, 59 \%$ and $51 \%$ in the first, second, third, fourth and fifth years, respectively.

There was a trend for older women to have higher adherence $(P<0.0001)$ and patients were more likely to adhere to the treatment therapy if they started on AI $(P=0.001)$, but there was no difference by other characteristics (Table 2).

There were $576(17 \%)$ patients with low adherence of whom 266 (46\%) died during follow-up compared with the 2785 (83\%) patients with high adherence of whom 928 (33\%) died. Using a multivariate model adjusting for all factors, patients with low adherence of endocrine therapy were at significantly higher risk of all-cause mortality $(\mathrm{HR}=1.199 ; 95 \% \mathrm{CI}=1.030,1.396 ; P=0.019)$. A separate multivariate model adjusting for the same factors, but looking at cumulative years of adherence that showed patients with less than 3 years good adherence (i.e., $>80 \%$ ), were at increased risk of death compared with patients having 5 years of good adherence. Increasing age, tumour stage, nodal stage, tumour grade, presence of metastases, negative ER status and a delay in starting medication for 6 months or more also increased the risk of all-cause mortality (Table 3 ).

There were 740 patients reported as having breast cancer as the cause of death, 137 (33\%) in patients with low adherence and 607 $(21 \%)$ in those with high adherence. There was no significant increased risk of breast cancer death by level of adherence over the entire treatment period using multivariate models. However, a separate model suggested patients with 5 years of cumulative adherence were at lower risk of breast cancer death than those with 3 years high adherence or less. Increasing age, tumour stage, nodal stage, tumour grade, presence of metastases, negative ER status and
Table 2. Descriptive statistics of adjuvant endocrine cohort $(n=3361)$ by adherence

\begin{tabular}{|l|c|c|l|}
\hline & $\begin{array}{c}\text { Low } \\
\text { adherence } \\
(\%)\end{array}$ & $\begin{array}{c}\text { High } \\
\text { adherence } \\
(\%)\end{array}$ & $\begin{array}{c}\chi^{2} \text {-test (Pearson's, } \\
\text { d.f., P-value) }\end{array}$ \\
\hline Numbers & $576(17)$ & $2785(83)$ & - \\
\hline
\end{tabular}

\section{SIMD quintile}

\begin{tabular}{|l|c|c|c|}
\hline 1 & $89(16)$ & $364(13)$ & $9.7 ; 4 ; 0.044$ \\
2 & $88(15)$ & $387(14)$ & - \\
3 & $109(19)$ & $436(16)$ & - \\
4 & $164(29)$ & $932(34)$ & - \\
5 & $120(21)$ & $618(23)$ & - \\
Unknown & 6 & 48 & - \\
\hline
\end{tabular}

\begin{tabular}{|c|c|c|c|}
\hline \multicolumn{4}{|c|}{ Age group } \\
\hline$<40$ & $45(8)$ & $88(3)$ & $41.8 ; 5 ;<0.0001$ \\
\hline $40-49$ & $94(16)$ & $363(13)$ & - \\
\hline $50-59$ & $149(26)$ & $643(23)$ & - \\
\hline $60-69$ & $114(20)$ & $657(24)$ & - \\
\hline 70-79 & $90(16)$ & $579(21)$ & - \\
\hline $80+$ & $84(14)$ & 455 (16) & - \\
\hline
\end{tabular}

\section{Charlson's index}

\begin{tabular}{|l|l|r|c|}
\hline $0-2$ & $179(31)$ & $833(30)$ & $0.4 ; 2 ; 0.809$ \\
$3-5$ & $227(39)$ & $1098(39)$ & - \\
$6+$ & $170(30)$ & $854(31)$ & - \\
\hline
\end{tabular}

Tumour stage

\begin{tabular}{|l|c|c|c|}
\hline 1 & $152(44)$ & $640(43)$ & $6.8 ; 3 ; 0.077$ \\
2 & $111(33)$ & $541(36)$ & - \\
3 & $36(11)$ & $101(7)$ & - \\
4 & $43(12)$ & $206(14)$ & - \\
Unknown & 234 & 1297 & - \\
\hline
\end{tabular}

Clinical nodes

\begin{tabular}{|l|c|c|c|}
\hline 0 & $353(80)$ & $1591(82)$ & $0.5 ; 2 ; 0.785$ \\
1 & $73(17)$ & $298(15)$ & - \\
2 & $12(3)$ & $52(3)$ & - \\
Unknown & 138 & 844 & - \\
\hline
\end{tabular}

\section{Clinical metastases}

\begin{tabular}{|l|c|c|c|}
\hline No & $410(94)$ & $1796(96)$ & $1 ; 1 ; 0.320$ \\
Yes & $24(6)$ & $83(4)$ & - \\
Unknown & 142 & 906 & - \\
\hline
\end{tabular}

\section{Tumour grade}

\begin{tabular}{|l|c|c|c|}
\hline 1 & $70(17)$ & $325(15)$ & $2.5 ; 2 ; 0.282$ \\
2 & $201(48)$ & $1139(52)$ & - \\
3 & $150(35)$ & $731(33)$ & - \\
Unknown & 155 & 590 & - \\
\hline
\end{tabular}

ER status

\begin{tabular}{|l|c|c|c|}
\hline Positive & $362(90)$ & $1958(92)$ & $3.9 ; 1 ; 0.050$ \\
Negative & $42(10)$ & $159(8)$ & \\
Unknown & 172 & 668 & \\
\hline
\end{tabular}

Abbreviations: $\mathrm{ER}=$ oestrogen receptor; $\mathrm{SIMD}=$ Scottish Index of Multiple Deprivation.

a delay in starting medication for 6 months or more also increased the risk of breast cancer mortality (Table 4 ).

There were a total of 552 recurrences reported from case note reviews, 123 (30\%) in patients with low adherence and $429(15 \%)$ 
Table 3. Multivariate association between adherence and all-cause mortality adjusted for covariates

Unadjusted

Adjusted

\begin{tabular}{|l|l|l|l|l|l|l|}
\hline Predictor & HR & $95 \% \mathrm{Cl}$ & $\boldsymbol{P}$-value & HR & $95 \% \mathrm{Cl}$ & $\boldsymbol{P}$-value
\end{tabular}

\section{Adherence}

\begin{tabular}{l|c|c|c|c|c|}
\hline 0.8 & 1 & - & - & 1 & - \\
$<0.8$ & 1.099 & $0.943-1.281$ & 0.226 & 1.199 & 0.019
\end{tabular}

\section{Cumulative adherence $^{a}$}

\begin{tabular}{|c|c|c|c|c|c|c|}
\hline$\geqslant 5$ & 1 & - & - & 1 & - & - \\
\hline 4 & 1.138 & $0.871-1.486$ & 0.345 & 1.390 & $1.081-1.786$ & 0.010 \\
\hline 3 & 1.754 & $1.353-2.274$ & $<0.0001$ & 2.192 & $1.708-2.813$ & $<0.0001$ \\
\hline 2 & 3.551 & $2.755-4.576$ & $<0.0001$ & 4.686 & $3.689-5.952$ & $<0.0001$ \\
\hline$\leqslant 1$ & 5.595 & $4.426-7.071$ & $<0.0001$ & 8.147 & 6.479-10.244 & $<0.0001$ \\
\hline
\end{tabular}

Menopause

\begin{tabular}{|l|c|c|c|c|c|c|}
\hline Pre & 1 & - & - & 1 & - \\
Peri & 0.235 & $0.086-0.642$ & 0.005 & 0.204 & $0.075-0.559$ \\
Post & 1.937 & $1.526-2.459$ & $<0.0001$ & 0.548 & $0.414-0.724$ \\
Unknown & 1.810 & $1.425-2.299$ & $<.0001$ & 0.568 & $0.431-0.749$
\end{tabular}

Age

\begin{tabular}{|c|c|c|c|c|c|c|}
\hline+1 year & 1.051 & $1.046-1.055$ & $<0.0001$ & 1.052 & $1.047-1.058$ & $<0.0001$ \\
\hline \multicolumn{7}{|c|}{ SIMD quintile } \\
\hline 1 & 1 & - & - & 1 & - & - \\
\hline 2 & 1.041 & $0.848-1.278$ & 0.702 & 1.051 & $0.854-1.294$ & 0.636 \\
\hline 3 & 0.945 & $0.772-1.156$ & 0.581 & 0.933 & $0.761-1.145$ & 0.508 \\
\hline 4 & 0.878 & $0.734-1.051$ & 0.157 & 0.930 & $0.775-1.116$ & 0.433 \\
\hline 5 & 0.752 & $0.617-0.915$ & 0.004 & 0.819 & $0.671-0.999$ & 0.049 \\
\hline Unknown & 1.486 & $0.984-2.243$ & 0.060 & 1.453 & $0.955-2.210$ & 0.081 \\
\hline
\end{tabular}

Tumour stage

\begin{tabular}{|l|c|c|c|c|c}
\hline T1 & 1 & - & - & 1 & - \\
T2 & 2.140 & $1.785-2.566$ & $<0.0001$ & 1.458 & $1.212-1.756$ \\
T3 & 3.731 & $2.885-4.826$ & $<0.0001$ & 2.183 & $1.659-2.872$ \\
T4 & 4.532 & $3.675-5.589$ & $<0.0001$ & 1.616 & $1.274-2.050$ \\
Unknown & 1.576 & $1.329-1.868$ & $<0.0001$ & 1.146 & $0.944-1.392$ \\
\hline
\end{tabular}

\section{Nodal stage}

\begin{tabular}{|l|c|c|r|r|r}
\hline N0 & 1 & - & - & 1 & - \\
N1 & 2.741 & $2.343-3.206$ & $<0.0001$ & 1.665 & $1.404-1.975$ \\
N2 & 3.516 & $2.587-4.777$ & $<0.0001$ & 1.518 & $1.079-2.135$ \\
Unknown & 1.303 & $1.137-1.492$ & $<0.0001$ & 1.264 & 0.017 \\
\hline
\end{tabular}

\section{Metastases stage}

\begin{tabular}{|c|c|c|c|c|c|c|}
\hline $\begin{array}{l}\text { M0 } \\
\text { M1 } \\
\text { Unknown }\end{array}$ & $\begin{array}{c}1 \\
4.439 \\
1.133\end{array}$ & $\begin{array}{c}- \\
3.539-5.566 \\
0.995-1.290\end{array}$ & $\begin{array}{c}- \\
<0.0001 \\
0.059\end{array}$ & $\begin{array}{c}1 \\
1.835 \\
0.758\end{array}$ & $\begin{array}{c}-\overline{1} \\
1.412-2.385 \\
0.597-0.961\end{array}$ & $\begin{array}{c}-\overline{0} \\
<0.0001 \\
0.022\end{array}$ \\
\hline \multicolumn{7}{|c|}{ Tumour grade } \\
\hline $\begin{array}{l}\text { G1 } \\
\text { G2 } \\
\text { G3 } \\
\text { Unknown }\end{array}$ & $\begin{array}{c}1 \\
1.639 \\
2.419 \\
3.234\end{array}$ & $\begin{array}{c}- \\
1.277-2.105 \\
1.880-3.112 \\
2.532-4.130\end{array}$ & $\begin{array}{l}- \\
<0.0001 \\
<0.0001 \\
<0.0001\end{array}$ & $\begin{array}{c}1 \\
1.300 \\
1.789 \\
1.788\end{array}$ & $\begin{array}{c}- \\
1.009-1.674 \\
1.379-2.321 \\
1.355-2.360\end{array}$ & $\begin{array}{c}-\overline{-} \\
0.042 \\
<0.0001 \\
<0.0001\end{array}$ \\
\hline \multicolumn{7}{|c|}{ ER status } \\
\hline $\begin{array}{l}\text { Positive } \\
\text { Negative } \\
\text { Unknown }\end{array}$ & $\begin{array}{c}1 \\
2.681 \\
1.771\end{array}$ & $\begin{array}{c}- \\
2.225-3.230 \\
1.565-2.005\end{array}$ & $\begin{array}{l}- \\
<0.0001 \\
<0.0001\end{array}$ & $\begin{array}{c}1 \\
2.019 \\
1.444\end{array}$ & $\begin{array}{c}- \\
1.643-2.481 \\
1.214-1.717\end{array}$ & $\begin{array}{c}-\overline{0} \\
<0.0001 \\
<0.0001\end{array}$ \\
\hline \multicolumn{7}{|c|}{ Treatment therapy } \\
\hline $\begin{array}{l}\text { Tam } \\
\text { Al } \\
\text { Mix }\end{array}$ & $\begin{array}{c}1 \\
1.846 \\
1.378\end{array}$ & $\begin{array}{c}- \\
1.466-2.325 \\
1.209-1.571\end{array}$ & $\begin{array}{l}- \\
<0.0001 \\
<0.0001\end{array}$ & $\begin{array}{c}1 \\
1.489 \\
1.231\end{array}$ & $\begin{array}{c}- \\
1.164-1.906 \\
1.071-1.416\end{array}$ & $\begin{array}{c}-\overline{0} \\
0.002 \\
0.003\end{array}$ \\
\hline
\end{tabular}




\section{Table 3. (Continued)}

\begin{tabular}{|c|c|c|c|c|c|c|}
\hline & \multicolumn{3}{|c|}{ Unadjusted } & \multicolumn{3}{|c|}{ Adjusted } \\
\hline Predictor & HR & $95 \% \mathrm{Cl}$ & $\boldsymbol{P}$-value & HR & $95 \% \mathrm{Cl}$ & $\boldsymbol{P}$-value \\
\hline \multicolumn{7}{|c|}{ Charlson's index } \\
\hline $\begin{array}{l}0-2 \\
3-5 \\
6+\end{array}$ & $\begin{array}{c}1 \\
0.959 \\
0.996\end{array}$ & $\begin{array}{c}- \\
0.836-1.100 \\
0.862-1.150\end{array}$ & $\begin{array}{l}-\overline{0} \\
0.950 \\
0.951\end{array}$ & $\begin{array}{c}1 \\
1.037 \\
1.073\end{array}$ & $\begin{array}{c}- \\
0.902-1.193 \\
0.927-1.242\end{array}$ & $\begin{array}{c}- \\
0.607 \\
0.343\end{array}$ \\
\hline \multicolumn{7}{|l|}{ Delay } \\
\hline $\begin{array}{l}\leqslant 6 \text { months } \\
>6 \text { months }\end{array}$ & $\begin{array}{c}1 \\
1.040\end{array}$ & $\frac{-}{0.880-1.228}$ & $\overline{0} .648$ & $\begin{array}{c}1 \\
1.430\end{array}$ & $\frac{-}{1.189-1.721}$ & $\begin{array}{c}- \\
<0.0001\end{array}$ \\
\hline
\end{tabular}

Table 4. Multivariate association between adherence and breast-cancer mortality adjusted for covariates

Unadjusted

\begin{tabular}{|c|c|c|c|c|c|c|}
\hline Predictor & $\mathrm{HR}$ & $95 \% \mathrm{Cl}$ & $\boldsymbol{P}$-value & HR & $95 \% \mathrm{Cl}$ & $P$-value \\
\hline \multicolumn{7}{|c|}{ Adherence } \\
\hline $\begin{array}{l}\geqslant 0.8 \\
<0.8\end{array}$ & $\begin{array}{c}1 \\
1.297\end{array}$ & $\frac{-}{1.077-1.563}$ & $\overline{0.006}$ & $\begin{array}{c}1 \\
1.150\end{array}$ & $\begin{array}{c}- \\
0.950-1.393\end{array}$ & $\overline{0.152}$ \\
\hline \multicolumn{7}{|c|}{ Cumulative adherence $^{a}$} \\
\hline $\begin{array}{l}\geqslant 5 \\
4 \\
3 \\
2 \\
\leqslant 1\end{array}$ & $\begin{array}{c}1 \\
1.029 \\
2.100 \\
4.849 \\
8.635\end{array}$ & $\begin{array}{c}- \\
0.677-1.564 \\
1.426-3.093 \\
3.343-7.034 \\
6.092-12.239\end{array}$ & $\begin{array}{c}-\overline{-} \\
0.894 \\
<0.0001 \\
<0.0001 \\
<0.0001\end{array}$ & $\begin{array}{c}1 \\
1.655 \\
2.963 \\
6.823 \\
13.374\end{array}$ & $\begin{array}{c}- \\
1.119-2.449 \\
2.023-4.339 \\
4.747-9.807 \\
9.414-19.000\end{array}$ & $\begin{array}{c}-\overline{-} \\
0.012 \\
<0.0001 \\
<0.0001 \\
<0.0001\end{array}$ \\
\hline \multicolumn{7}{|c|}{ Menopause } \\
\hline $\begin{array}{l}\text { Pre } \\
\text { Peri } \\
\text { Post } \\
\text { Unknown }\end{array}$ & $\begin{array}{c}1 \\
0.295 \\
1.149 \\
1.159\end{array}$ & $\begin{array}{c}- \\
0.199-0.730 \\
0.895-1.475 \\
0.902-1.488\end{array}$ & $\begin{array}{l}-\overline{-} \\
0.008 \\
0.277 \\
0.248\end{array}$ & $\begin{array}{c}1 \\
0.331 \\
0.631 \\
0.623\end{array}$ & $\begin{array}{c}- \\
0.133-0.821 \\
0.465-0.856 \\
0.463-0.840\end{array}$ & $\begin{array}{c}-\overline{0} \\
0.017 \\
0.003 \\
0.002\end{array}$ \\
\hline \multicolumn{7}{|l|}{ Age } \\
\hline 1 year & 1.024 & $1.019-1.030$ & $<0.0001$ & 1.023 & $1.016-1.030$ & $<0.0001$ \\
\hline \multicolumn{7}{|c|}{ SIMD quintile } \\
\hline $\begin{array}{l}1 \\
2 \\
3 \\
4 \\
5 \\
\text { Unknown }\end{array}$ & $\begin{array}{c}1 \\
1.051 \\
1.008 \\
0.906 \\
0.829 \\
1.209\end{array}$ & $\begin{array}{c}- \\
0.806-1.369 \\
0.779-1.305 \\
0.719-1.142 \\
0.646-1.064 \\
0.679-2.152\end{array}$ & $\begin{array}{c}- \\
0.715 \\
0.949 \\
0.405 \\
0.141 \\
0.518\end{array}$ & $\begin{array}{c}1 \\
1.047 \\
1.092 \\
0.960 \\
0.936 \\
1.473\end{array}$ & $\begin{array}{c}- \\
0.802-1.368 \\
0.842-1.416 \\
0.760-1.213 \\
0.727-1.206 \\
0.820-2.646\end{array}$ & $\begin{array}{c}-\overline{-} \\
0.736 \\
0.509 \\
0.733 \\
0.611 \\
0.195\end{array}$ \\
\hline \multicolumn{7}{|c|}{ Tumour stage } \\
\hline $\begin{array}{l}\text { T1 } \\
\text { T2 } \\
\text { T3 } \\
\text { T4 } \\
\text { Unknown }\end{array}$ & $\begin{array}{c}1 \\
3.229 \\
7.115 \\
7.265 \\
2.198 \\
\end{array}$ & $\begin{array}{c}- \\
2.493-4.183 \\
5.166-9.801 \\
5.476-9.638 \\
1.715-2.819\end{array}$ & $\begin{array}{c}-\overline{-} \\
<0.0001 \\
<0.0001 \\
<0.0001 \\
<0.0001\end{array}$ & $\begin{array}{c}1 \\
2.228 \\
3.493 \\
2.309 \\
1.399 \\
\end{array}$ & $\begin{array}{c}- \\
1.711-2.903 \\
2.481-4.920 \\
1.673-3.186 \\
1.060-1.847\end{array}$ & $\begin{array}{c}-\overline{-} \\
<0.0001 \\
<0.0001 \\
<0.0001 \\
0.018\end{array}$ \\
\hline \multicolumn{7}{|c|}{ Nodal stage } \\
\hline $\begin{array}{l}\text { N0 } \\
\text { N1 } \\
\text { N2 } \\
\text { Unknown }\end{array}$ & $\begin{array}{c}1 \\
3.770 \\
4.808 \\
1.466\end{array}$ & $\begin{array}{c}- \\
3.131-4.539 \\
3.392-6.816 \\
1.228-1.749\end{array}$ & $\begin{array}{c}-\overline{-} \\
<0.0001 \\
<0.0001 \\
<0.0001\end{array}$ & $\begin{array}{c}1 \\
1.967 \\
1.508 \\
1.349\end{array}$ & $\begin{array}{c}- \\
1.608-2.407 \\
1.013-2.247 \\
0.973-1.870\end{array}$ & $\begin{array}{c}-\overline{-} \\
<0.0001 \\
0.043 \\
0.072\end{array}$ \\
\hline
\end{tabular}


Table 4. (Continued)

\begin{tabular}{|c|c|c|c|c|c|c|}
\hline & \multicolumn{3}{|c|}{ Unadjusted } & \multicolumn{3}{|c|}{ Adjusted } \\
\hline Predictor & HR & $95 \% \mathrm{Cl}$ & $\boldsymbol{P}$-value & HR & $95 \% \mathrm{Cl}$ & $\boldsymbol{P}$-value \\
\hline \multicolumn{7}{|c|}{ Metastases stage } \\
\hline $\begin{array}{l}\text { M0 } \\
\text { M1 } \\
\text { Unknown } \\
-\end{array}$ & $\begin{array}{c}1 \\
6.268 \\
1.255 \\
-\end{array}$ & $\begin{array}{c}- \\
4.894- \\
8.027 \\
1.066-1.479\end{array}$ & $\begin{array}{c}- \\
<0.0001 \\
0.007 \\
-\end{array}$ & $\begin{array}{c}1 \\
2.119 \\
0.938 \\
-\end{array}$ & $\begin{array}{c}- \\
1.574-2.853 \\
0.699-1.261 \\
-\end{array}$ & $\begin{array}{c}- \\
<0.0001 \\
0.673 \\
-\end{array}$ \\
\hline \multicolumn{7}{|c|}{ Tumour grade } \\
\hline $\begin{array}{l}\text { G1 } \\
\text { G2 } \\
\text { G3 } \\
\text { Unknown }\end{array}$ & $\begin{array}{c}1 \\
3.037 \\
5.480 \\
6.268\end{array}$ & $\begin{array}{c}- \\
1.990-4.635 \\
3.599-8.344 \\
4.128-9.518\end{array}$ & $\begin{array}{c}-\overline{-} \\
<0.0001 \\
<0.0001 \\
<0.0001\end{array}$ & $\begin{array}{c}1 \\
2.089 \\
3.274 \\
3.293\end{array}$ & $\begin{array}{c}- \\
1.364-3.198 \\
2.133-5.026 \\
2.097-5.171\end{array}$ & $\begin{array}{c}-\overline{0} \\
0.001 \\
<0.0001 \\
<0.0001\end{array}$ \\
\hline \multicolumn{7}{|l|}{ ER status } \\
\hline $\begin{array}{l}\text { Positive } \\
\text { Negative } \\
\text { Unknown }\end{array}$ & $\begin{array}{c}1 \\
3.113 \\
1.758\end{array}$ & $\begin{array}{c}- \\
2.489-3.894 \\
1.501-2.061\end{array}$ & $\begin{array}{c}-\overline{-} \\
<0.0001 \\
<0.0001\end{array}$ & $\begin{array}{c}1 \\
2.350 \\
1.403\end{array}$ & $\begin{array}{c}- \\
1.831-3.017 \\
1.119-1.759\end{array}$ & $\begin{array}{c}-\overline{-} \\
<0.0001 \\
0.003\end{array}$ \\
\hline \multicolumn{7}{|c|}{ Treatment therapy } \\
\hline $\begin{array}{l}\text { Tam } \\
\text { Al } \\
\text { Mix }\end{array}$ & $\begin{array}{c}1 \\
2.350 \\
2.395\end{array}$ & $\begin{array}{c}- \\
1.785-3.093 \\
2.053-2.793\end{array}$ & $\begin{array}{c}-\overline{0} \\
<0.0001 \\
<0.0001\end{array}$ & $\begin{array}{c}1 \\
2.133 \\
2.091\end{array}$ & $\begin{array}{c}- \\
1.583-2.874 \\
1.773-2.467\end{array}$ & $\begin{array}{c}-\overline{-} \\
<0.0001 \\
<0.0001\end{array}$ \\
\hline \multicolumn{7}{|c|}{ Charlson's index } \\
\hline $\begin{array}{l}0-2 \\
3-5 \\
6+\end{array}$ & $\begin{array}{c}1 \\
0.974 \\
1.012\end{array}$ & $\begin{array}{c}- \\
0.818-1.160 \\
0.842-1.215\end{array}$ & $\begin{array}{c}-\overline{0} \\
0.767 \\
0.901\end{array}$ & $\begin{array}{c}1 \\
1.089 \\
1.132\end{array}$ & $\begin{array}{c}-\overline{-} \\
0.912-1.301 \\
0.939-1.364\end{array}$ & $\begin{array}{c}-\overline{0} \\
0.345 \\
0.193\end{array}$ \\
\hline \multicolumn{7}{|l|}{ Delay } \\
\hline $\begin{array}{l}\leqslant 6 \text { months } \\
>6 \text { months }\end{array}$ & $\begin{array}{c}1 \\
1.411\end{array}$ & $\frac{-}{1.168-1.703}$ & $\begin{array}{c}- \\
<0.0001\end{array}$ & $\begin{array}{c}1 \\
1.305\end{array}$ & $\begin{array}{c}- \\
1.054-1.615\end{array}$ & $\overline{0}-\overline{014}$ \\
\hline
\end{tabular}

in patients with high adherence. There was no significant difference in risk of recurrence for patients with low adherence over the entire treatment period after adjusting for other factors. However, fewer cumulative years of high adherence was associated with increased risk of recurrence, although this was only significant comparing 5 years against 3 or less.

Tumour stage, nodal stage, tumour grade, presence of metastases or negative ER status and a delay in starting medication of 6 months or more increased the risk of recurrence (Table 5).

The use of AIs as endocrine therapy either alone or in sequence with tamoxifen was associated with an increased risk of all-cause mortality (AIs: $1.489,95 \% \mathrm{CI}=1.164-1.906$; combination: 1.231 , $95 \% \mathrm{CI}=1.071-1.416$ ), breast cancer-specific mortality (AIs: 2.133; $95 \% \quad \mathrm{CI}=1.583-2.874$; combination: $2.091, \quad 95 \%$ $\mathrm{CI}=1.773-2.467$ ) and recurrence (AIs: $1.693,95 \% \mathrm{CI}=1.084-$ 2.644; combination: $4.606,95 \% \mathrm{CI}=3.792-5.594)$.

To examine any effect based on year of diagnosis, the three models were repeated with this variable added, but there was little difference on the reported effect of adherence (for all-cause mortality low adherence $\mathrm{HR}=1.184 ; 95 \% \mathrm{CI}=1.010-1.389$; $P=0.037$ ) and year of diagnosis was not a significant predictor. However, dichotomising year of diagnosis to 1993 to 2001 or 2002 onwards did show an effect (for all-cause mortality 1993-2001 vs 2002 onwards; $\mathrm{HR}=1.316 ; 95 \% \mathrm{CI}=1.022-1.696 ; P=0.033$ ), but there was again no difference on the reported effect of adherence.

\section{DISCUSSION}

This study reports that low adherence is associated with increased all-cause mortality, and that the longer a patient is adherent, the less their risk of all-cause and breast cancer-specific mortality and recurrence. Our findings also confirm that although overall adherence to daily oral adjuvant endocrine therapy in the community setting is initially high, as time progresses patients become less adherent. Many women do not take their adjuvant therapy for the recommended 5 -year period, with $\sim 52 \%$ of those who began having discontinued treatment by 5 years.

Using AIs alone or sequentially with tamoxifen was associated with poorer outcomes in post-menopausal women, reflecting the local management policy in practice at the time of the study, where women at higher risk of recurrence following initial treatment of the primary breast cancer were prescribed AIs, whereas women perceived to be at lower risk of recurrence were prescribed tamoxifen. A delay in starting treatment of 6 months or more was also associated with poorer outcomes, but this would again reflect higher-stage disease and additional adjuvant therapies (adjuvant systemic chemotherapy and post-operative radiotherapy) following primary surgery. However, even after adjusting for these factors and tumour characteristics on presentation, high adherence was associated with better outcomes. Comorbidity was not associated 
Table 5. Multivariate association between adherence and recurrence adjusted for covariates

Unadjusted

Adjusted

\begin{tabular}{|c|c|c|c|c|c|c|}
\hline Predictor & HR & $95 \% \mathrm{Cl}$ & $P$-value & HR & $95 \% \mathrm{Cl}$ & $\boldsymbol{P}$-value \\
\hline \multicolumn{7}{|c|}{ Adherence } \\
\hline $\begin{array}{l}\geqslant 0.8 \\
<0.8\end{array}$ & $\begin{array}{c}1 \\
1.689\end{array}$ & $1.371-2.082$ & $<0.0001$ & $\begin{array}{c}1 \\
1.219\end{array}$ & $0.970-1.531$ & 0.089 \\
\hline \multicolumn{7}{|c|}{ Cumulative adherence $^{a}$} \\
\hline $\begin{array}{l}\geqslant 5 \\
4 \\
3 \\
2 \\
\leqslant 1\end{array}$ & \begin{tabular}{c|}
1 \\
1.079 \\
1.730 \\
3.357 \\
3.353
\end{tabular} & $\begin{array}{c}- \\
0.724-1.607 \\
1.179-2.539 \\
2.304-4.889 \\
2.329-4.827\end{array}$ & $\begin{aligned}- & 0.708 \\
< & 0.005 \\
< & 0.0001 \\
< & 0.0001\end{aligned}$ & $\begin{array}{c}1 \\
1.653 \\
2.667 \\
4.567 \\
5.860\end{array}$ & $\begin{array}{c}- \\
1.136-2.404 \\
1.838-3.868 \\
3.160-6.601 \\
4.074-8.429\end{array}$ & $\begin{array}{l}- \\
0.009 \\
<0.0001 \\
<0.0001 \\
<0.0001\end{array}$ \\
\hline
\end{tabular}

\section{Menopause}

\begin{tabular}{|l|c|c|}
\hline Pre & 1 & - \\
Peri & 0.292 & $0.128-0.667$ \\
Post & 0.787 & $0.618-1.001$ \\
Unknown & 0.428 & $0.329-0.557$
\end{tabular}

$0.329-0.557$

\begin{tabular}{c|c}
- & 1 \\
0.004 & 0.341 \\
0.051 & 0.812 \\
$<0.0001$ & 0.400
\end{tabular}

-
$0.148-0.785$
$0.592-1.114$

$0.592-1.114$
$0.291-0.551$

0.011

0.197

$<0.0001$

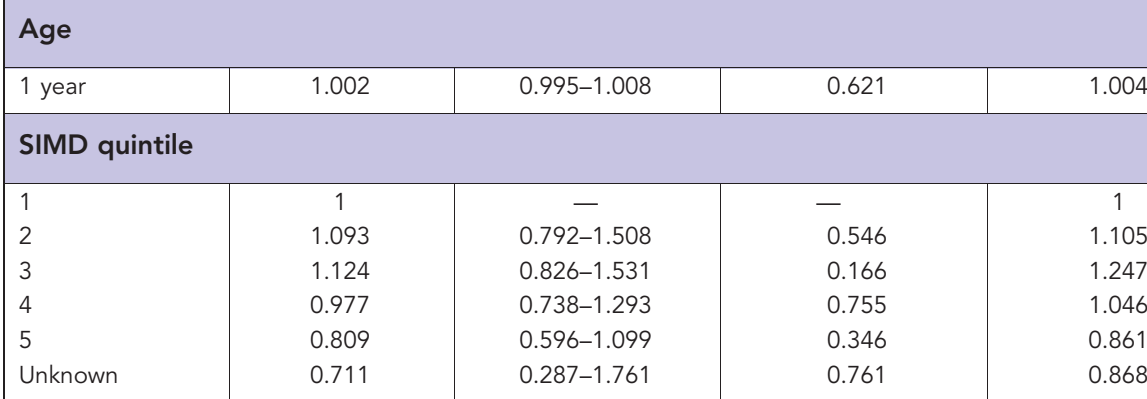

\section{Tumour stage}

\begin{tabular}{l|c}
\hline T1 & 1 \\
T2 & 2.643 \\
T3 & 4.697 \\
T4 & 4.540 \\
Unknown & 1.609 \\
\hline
\end{tabular}

1
2.643
4.697
4.540
1.609

$-$

-
$<0.0001$
$<0.0001$
$<0.0001$
$<0.0001$

-
$2.003-3.488$
$3.212-6.870$
$3.252-6.337$
$1.231-2.103$

1
2.264
2.514
1.980
1.284

\begin{tabular}{|l|c|c|c|c|c|c|}
\hline N0 & 1 & - & - & 1 & - & - \\
N1 & 3.131 & $2.502-3.918$ & $<0.0001$ & 1.966 & $1.547-2.498$ & $<0.0001$ \\
N2 & 3.745 & $2.377-5.901$ & $<0.0001$ & 1.712 & $1.024-2.862$ & 0.040 \\
Unknown & 0.965 & $0.771-1.208$ & 0.756 & 1.378 & $0.943-2.015$ & 0.098 \\
\hline
\end{tabular}

-
$0.799-1.529$
$0.913-1.702$
$0.788-1.389$
$0.631-1.175$
$0.347-2.168$

\section{Nodal stage}

\section{Metastases stage}

\section{MO}

M1

Unknown

\section{Tumour grade}

\begin{tabular}{|l|r}
\hline G1 & 1 \\
G2 & 1.948 \\
G3 & 3.867 \\
Unknown & 2.307
\end{tabular}

Unknown

\section{ER status}

Positive

Negative

Unknown

\begin{abstract}
0.965
\end{abstract}
$0.943-2.015$

-

$<0.0001$

$<0.0001$

$<0.0001$

0.097

$1.352-2.902$

$0.956-1.725$
0.098

(

1
3.212
0.834

0.834

1
1.948
3.867
2.307

2.156-4.785

0.676-1.029

\section{$<0.0001$}

0.090

1
1.166
0.954

0.954
-

$0.738-1.843$

$0.678-1.343$

0.510

0.788 
Table 5. (Continued)

\begin{tabular}{|c|c|c|c|c|c|c|}
\hline & \multicolumn{3}{|c|}{ Unadjusted } & \multicolumn{3}{|c|}{ Adjusted } \\
\hline Predictor & HR & $95 \% \mathrm{Cl}$ & $\boldsymbol{P}$-value & HR & $95 \% \mathrm{Cl}$ & $P$-value \\
\hline \multicolumn{7}{|c|}{ Treatment therapy } \\
\hline $\begin{array}{l}\text { Tam } \\
\text { Al } \\
\text { Mix }\end{array}$ & $\begin{array}{c}1 \\
1.922 \\
4.893\end{array}$ & $\begin{array}{c}- \\
1.258-2.937 \\
4.077-5.873\end{array}$ & $\begin{aligned}- & 0.003 \\
< & 0.0001\end{aligned}$ & $\begin{array}{c}1 \\
1.693 \\
4.606\end{array}$ & $\begin{array}{c}- \\
1.084-2.644 \\
3.792-5.594\end{array}$ & $\begin{aligned}- & 0.021 \\
< & 0.0001\end{aligned}$ \\
\hline \multicolumn{7}{|c|}{ Charlson's index } \\
\hline $\begin{array}{l}0-2 \\
3-5 \\
6+\end{array}$ & $\begin{array}{c}1 \\
1.028 \\
1.048 \\
\end{array}$ & $\begin{array}{c}-- \\
0.833-1.270 \\
0.839-1.309\end{array}$ & $\begin{array}{l}- \\
0.795 \\
0.682\end{array}$ & $\begin{array}{c}1 \\
1.131 \\
1.165\end{array}$ & $\begin{array}{c}- \\
0.913-1.401 \\
0.929-1.462\end{array}$ & $\begin{array}{l}- \\
0.260 \\
0.185\end{array}$ \\
\hline \multicolumn{7}{|l|}{ Delay } \\
\hline $\begin{array}{l}\leqslant 6 \text { months } \\
>6 \text { months }\end{array}$ & $\begin{array}{c}1 \\
1.864\end{array}$ & $\begin{array}{c}- \\
1.509-2.303\end{array}$ & $\begin{array}{l}- \\
<0.0001\end{array}$ & $\begin{array}{c}1 \\
1.360\end{array}$ & $\begin{array}{c}- \\
1.070-1.727\end{array}$ & $-\overline{0.012}$ \\
\hline
\end{tabular}

with worse outcomes within this analysis and socio-economic status was only significant, showing a reduction for the most affluent women compared with the most deprived for all-cause mortality.

Our results are consistent with other studies that have shown high discontinuation (in the range of $30-51 \%$ ) and poor adherence among women prescribed adjuvant endocrine therapy (McCowan et al, 2008; Hershman et al, 2010). Seventeen per cent of adjuvant endocrine therapy users had adherence below $80 \%$, similar to studies reported elsewhere, with younger women particularly at risk (Murthy et al, 2002; Partridge et al, 2003; McCowan et al, 2008; Hershman et al, 2010). Hence, the characteristics of adjuvant endocrine therapy use, adherence and persistence in this population are similar to those from other published work. This study has added to the existing literature, supporting the link between low adherence and increased all-cause mortality, but has added that longer periods of high adherence are associated with better outcomes.

In terms of generalisability, the study population comprised women who had a first incident breast cancer and started endocrine therapy, $70 \%$ of all women who were diagnosed with breast cancer in the region over the study period and so were highly representative.

Similar to other studies in the literature, this study has defined adherence to medication by examining the encashment of prescriptions, although not all aspects of adherence can be covered by this method of measurement and each technique has its own advantages and disadvantages (Osterberg and Blaschke, 2005; McCowan et al, 2008; Ho et al, 2009). Adherence to medications may be assessed by direct methods including directly observed therapy or indirect methods, such as pill counts. We cannot tell if patients actually took the medication, but breast cancer patients are probably motivated to be adherent, as reflected in the high levels of adherence reported by this and other studies (Osterberg and Blaschke, 2005; McCowan et al, 2008; Ho et al, 2009). Furthermore, direct methods are not practical for routine clinical use in a population-based study and tend to result in unrealistically high measures, as the subjects know they are being monitored.

We were unable to examine reasons for non-adherence, but a case note review of the women with an incidence of breast cancer between 1998 and 2007 had 382 women who discontinued medication, reporting they did so owing to side effects. Over half of the women, whose notes were reviewed, reported side effects due to endocrine therapy with differences by whether women were taking tamoxifen or AIs, which is reported elsewhere (McCowan and Thompson, 2012).

Adherence to therapy is positively associated with improved patient outcomes; thus, it would seem important that clinical strategies to improve adherence are developed. A recent review highlighted managing side effects, improved patient education and better communication between the patient and health-care professional among strategies that may work, but further work is needed (Hadji, 2010).

\section{CONCLUSION}

Women with low adherence are at greater risk of all-cause mortality, and fewer years of high adherence is also associated with increased breast cancer-specific mortality and recurrence. Nonpersistence with adjuvant endocrine therapy occurs in nearly half of patients prescribed treatment over the recommended 5-year course. Patients should be encouraged to take their medication on a daily basis for the full 5-year period, to ensure their best chance of survival.

\section{ACKNOWLEDGEMENTS}

We thank the Health Informatics Centre, University of Dundee, for anonymisation, record linkage and other procedural assistance in preparing the data set. $\mathrm{CMcC}$ and AT conceived the study and planned it with JD and PD. BM carried out the analysis with support from $\mathrm{CMcC}$ and $\mathrm{PD}$. All authors contributed to the writing of the paper. $\mathrm{CMcC}$ is the guarantor. The study was funded by Breast Cancer Campaign (grant 2008NovPR01).

\section{DISCLAIMER}

All data were fully anonymised and data use was compliant with the Health Informatics Centre research governance process, which are approved by the Tayside Committee on Medical Research 
Ethics and the Caldicott Guardian. All authors have completed the ICMJE uniform disclosure form at www.icmje.org/ coi_disclosure.pdf.

\section{REFERENCES}

Barron TI, Connolly R, Bennett K, Feely J, Kennedy MJ (2007) Early discontinuation of tamoxifen. Cancer 109(5): 832-839.

Bradburn MJ, Clark TG, Love SB, Altman DG (2003) Survival analysis part II: multivariate data analysis - an introduction to concepts and methods. $\mathrm{Br} J$ Cancer 89(3): 431-436.

Cuzick J, Sestak I, Baum M, Buzdar A, Howell A, Dowsett M, Forbes JF (2010) Effect of anastrozole and tamoxifen as adjuvant treatment for early-stage breast cancer: 10-year analysis of the ATAC trial. Lancet Oncol 11(12): 1135-1141.

Demissie S, Silliman RA, Lash TL (2001) Adjuvant tamoxifen: predictors of use, side effects, and discontinuation in older women. J Clin Oncol 19(2): $322-328$.

Dezentje VO, van Blijderveen NJC, Gelderblom H, Putter H, van Herk-Sukel MPP, Casparie MK, Egberts ACG, Nortier JWR, Guchelaar H-J (2010) Effect of concomitant CYP2D6 inhibitor use and tamoxifen adherence on breast cancer recurrence in early-stage breast cancer. J Clin Oncol 28(14): 2423-2429.

Early Breast Cancer Trialists' Collaborative Group (2011) Relevance of breast cancer hormone receptors and other factors to the efficacy of adjuvant tamoxifen: patient-level meta-analysis of randomised trials. Lancet 378(9793): 771-784.

Hadji P (2010) Improving compliance and persistence to adjuvant tamoxifen and aromatase inhibitor therapy. Crit Rev Oncol Hematol 73(2): $156-166$.

Hershman D, Shao T, Kushi L, Buono D, Tsai W, Fehrenbacher L, Kwan M, Gomez S, Neugut A (2011) Early discontinuation and non-adherence to adjuvant hormonal therapy are associated with increased mortality in women with breast cancer. Breast Cancer Res Treat 126(2): 529-537.

Hershman DL, Kushi LH, Shao T, Buono D, Kershenbaum A, Tsai W-Y, Fehrenbacher L, Lin Gomez S, Miles S, Neugut AI (2010) Early discontinuation and nonadherence to adjuvant hormonal therapy in a cohort of 8769 early-stage breast cancer patients. J Clin Oncol 28(27): 4120-4128.

Ho PM, Bryson CL, Rumsfeld JS (2009) Medication Adherence. Circulation 119(23): 3028-3035.

Ma AMT, Barone J, Wallis AE, Wu NJ, Garcia LB, Estabrook A, RosenbaumSmith SM, Tartter PI (2008) Noncompliance with adjuvant radiation, chemotherapy, or hormonal therapy in breast cancer patients. Am J Surg 196(4): 500-504.

McCowan C, Shearer J, Donnan PT, Dewar JA, Crilly M, Thompson AM, Fahey TP (2008) Cohort study examining tamoxifen adherence and its relationship to mortality in women with breast cancer. Br J Cancer 99(11): 1763-1768.

McCowan C, Thompson AM (2012) The importance of nonpharmacogenetic factors in endocrine therapy. Pharmacogenomics 13(6): 721-728.

Murthy V, Bharia G, Sarin R (2002) Tamoxifen non-compliance: does it matter? Lancet Oncol 3(11): 654.
Narod SA (2010) Compliance with tamoxifen in women with breast cancer and a BRCA1 or BRCA2 mutation. J Clin Oncol 28(33): e698-e699.

Osterberg L, Blaschke T (2005) Adherence to Medication. N Engl J Med 353(5): 487-497.

Owusu C, Buist DS, Field TS, Lash TL, Thwin SS, Geiger AM, Quinn VP, Frost F, Prout M, Yood MU, Wei F, Silliman RA, Owusu C, Buist DSM, Field TS, Lash TL, Thwin SS, Geiger AM, Quinn VP, Frost F, Prout M, Yood MU, Wei F, Silliman RA (2008) Predictors of tamoxifen discontinuation among older women with estrogen receptor-positive breast cancer. J Clin Oncol 26(4): 549-555.

Partridge AH, LaFountain A, Mayer E, Taylor BS, Winer E, Asnis-Alibozek A (2008) Adherence to initial adjuvant anastrozole therapy among women with early-stage breast cancer. J Clin Oncol 26(4): 556-562.

Partridge AH, Wang PS, Winer EP, Avorn J (2003) Nonadherence to adjuvant tamoxifen therapy in women with primary breast cancer. J Clin Oncol 21(4): 602-606.

Patridge A, Wang P, Winer E, Avorn J (2003) Nonadherence to adjuvant tamoxifen therapy in women with primary breast cancer. J Clin Oncol 21(4): 602-606.

Ruddy K, Mayer E, Partridge A (2009) Patient adherence and persistence with oral anticancer treatment. CA Cancer J Clin 59(1): 56-66.

Scottish Government (2012) Scottish Index of Multiple Deprivation: Scottish Government: Edinburgh.

Scottish Intercollegiate Guideline Network Guidelines (2005) Breast Cancer in Women: A National Clinical Guideline. Scottish Intercollegiate Guideline Network: Edinburgh.

SPSS Inc. (2008) SPSS Statistics for Windows. Version 17.0. SPSS Inc.: Chicago. van de Velde CJH, Rea D, Seynaeve C, Putter H, Hasenburg A, Vannetzel J-M, Paridaens R, Markopoulos C, Hozumi Y, Hille ETM, Kieback DG, Asmar L, Smeets J, Nortier JWR, Hadji P, Bartlett JMS, Jones SE (2011) Adjuvant tamoxifen and exemestane in early breast cancer (TEAM): a randomised phase 3 trial. Lancet 377(9762): 321-331.

van Herk-Sukel M, van de Poll-Franse L, Voogd A, Nieuwenhuijzen G, Coebergh J, Herings R (2010) Half of breast cancer patients discontinue tamoxifen and any endocrine treatment before the end of the recommended treatment period of 5 years: a population-based analysis. Breast Cancer Res Treat 122(3): 843-851.

Yood MU, Owusu C, Buist DS, Geiger AM, Field TS, Thwin SS, Lash TL, Prout MN, Wei F, Quinn VP, Frost FJ, Silliman RA, Yood MU, Owusu C, Buist DSM, Geiger AM, Field TS, Thwin SS, Lash TL, Prout MN, Wei F, Quinn VP, Frost FJ, Silliman RA (2008) Mortality impact of less-thanstandard therapy in older breast cancer patients. J Am Coll Surg 206(1): 66-75.

Ziller V, Kalder M, Albert US, Holzhauer W, Ziller M, Wagner U, Hadji P (2009) Adherence to adjuvant endocrine therapy in postmenopausal women with breast cancer. Ann Oncol 20(3): 431-436.

This work is published under the standard license to publish agreement. After 12 months the work will become freely available and the license terms will switch to a Creative Commons AttributionNonCommercial-Share Alike 3.0 Unported License. 